\title{
Gewalt diesseits, jenseits und am Rande des Staates. Ethnologische ${ }^{1}$ Positionen
}

\author{
Thomas Zitelmann
}

\begin{abstract}
Approaching anthropological research on violence means to approach a field of questions rather than of answers. It offers manifold perspectives on competing interpretations: comparative perspectives on a multitude of articulations between war and peace in small societies; on physiological and bodily practices of violence; on cultural, symbolic and cognitive patterns of violence; systemic channels and alternatives for violence derived from conflicts of interests; structural patterns with a perspective on relations between local violence; intermediate processes of inclusion/exclusion and over-arching political-economic relations. A strong current in recent research relates to the transposition of phenomena of violence formerly observed outside the sphere of "the state" to post-Cold War phenomena now observed under the simultaneous conditions of globalisation and fragmentation of statehood. Since anthropological research on violence derives its particular virtues from fieldwork, fresh ethnographic data and comparison, it breeds continuous antagonisms against reductive models. A continuous bone of contention here remains the issue of violence and cultural relativism.
\end{abstract}

Keywords: Ethnologie; Forschungsgeschichte; Gewalt; Kulturrelativismus; Randstaatlichkeit

\section{Prolog}

Der Reisende, der sich in Joseph Conrads Herz der Finsternis der Grauensherrschaft des Mr. Kurtz nähert, erfährt das Grauen zunächst nur durch Erzählungen und Gerüchte. Lange bevor der Held das Gewaltreich des Mr. Kurtz betritt, liegt latente Gewalt unsichtbar über dem Flusssystem, auf dem der Dampfer den Urwald durchschifft. Das Grauen liegt in einer abweisenden grünen Hölle, die voller Zeichen ist - die Natur, Flussschnellen und Sandbänke, der Nebel, die Geräusche, kryptische Nachrichten, die in verlassenen Dörfern gefunden werden, Schreie und Stille - „Good God! What is the meaning?“ (Con$\operatorname{rad} 1973,57)$

Aktuelle ethnologische Gewaltforschung hat Ähnlichkeiten mit der Bedeutungsfrage, die sich auch den Passagieren auf der Reise in das Reich des Mr. Kurtz stellt. Waren sich die Vertreter der älteren Forschung bis in das zweite Drittel des 20. Jahrhunderts zumindest phasenweise oder in Abgrenzung gegenüber widerstreitenden Richtungen noch einigermaßen sicher, wo Gewalt in biologischen, sozialen oder kulturellen Strukturen (nicht)

1 Unter dem Ethnologiebegriff sind hier auch Fachrichtungen wie die britische social anthropology, die amerikanische cultural anthropology und die französische anthropologie etc. zusammengefasst. 
zu verorten war, hat sich diese Sicherheit spätestens seit den 1980er Jahren aufgelöst. Versuchen wir die Gewalt in der Symbolik zu finden, die den Weg der Reise begleitet? Suchen wir sie in Gerüchten, Narrativen und versteckten Codes, die sprachlich nicht vermittelt werden können? Liegt die Gewalt in übergeordneten Strukturen (Kolonialismus, Imperialismus, Empire, Neoliberalismus, Globalisierung), denen Mr. Kurtz seinen Handelsposten im Dschungel verdankt? Ist Mr. Kurtz im System der Flussläufe, Dschungellabyrinthe und unzugänglicher Dörfer einer grünen Hölle gefangen, das sich seiner Psyche und seinem Körper einschreibt? Oder trägt Mr. Kurtz durch die Kanalisierung von Gewalt zu deren Vorhersehbarkeit und zur Vertrauensbildung in diesem System bei? Passt Mr. Kurtz sich den Gewaltgewohnheiten seiner Umwelt an und kommt in ihm dabei wieder ein ethologisch-ontologisch fixierter Aggressions-Gewalttrieb hervor? Organisiert der Handelsagent Kurtz hinter der Fassade von naturalisierend-terrorisierender Abschreckung einen effektiven und rational-operierenden Gewaltmarkt? Verweigert das Individuum Kurtz die intelligente und reziproke Kommunikation und macht sich der nihilistischen Gewalt schuldig? Wird das, was wir über den Gewalttäter Kurtz vom Hörensagen her wissen, seinen emischen Intentionen und den Bewertungen seiner direkten Umwelt gerecht? Welche Legitimitätsbegründungen liegen der Wertung von Kurtz Handlungen zu Grunde? Good God! What is the meaning of violence?

Leser, die in das Reich der ethnologischen Gewaltforschung eindringen wollen, müssen sich darauf einstellen, eher ein Reich vieler Fragen als eindeutiger Antworten zu betreten. Die methodisch über Feldforschung, Ethnographie und Vergleich vermittelte Betrachtung von Gewalt verhält sich gegenüber reduzierenden Modellbildungen sperrig. Vor diesem Hintergrund wird dieser Beitrag vier aktuelle Hauptrichtungen (Krieg und Frieden in einfachen Gesellschaften sowie systemische, kognitive und strukturelle Perspektiven) betrachten. Daran anschließend wird an die Geschichte der ethnologischen Gewaltforschung, die damit verbundenen Modellbildungen und ihre Kritiken erinnert. Zum Abschluss wird die Problematik von Kulturrelativismus und Gewaltanalyse diskutiert.

\section{Einführung in die ethnologische Gewaltforschung}

Ethnologische Zugänge zur Gewalt bilden auf den ersten Blick ein verwirrendes Bild konkurrierender Perspektiven: körperbezogen und physiologisch, symbolisch und kognitiv, systemisch mit starkem Bezug auf Konflikt oder strukturell mit Blick auf die Verbindungen zwischen lokalem Gewaltverhalten, intermediären Prozessen des Einschlusses/Ausschlusses und überdeterminierenden politisch-ökonomischen Zusammenhängen. Der Gewalt werden Wurzeln zugeschrieben, die abwechselnd in der menschlichen Natur oder in der sozialen und/oder kulturellen Reproduktion verortet werden. Gewalt bezieht sich auf externe Parameter, seien sie umweltbezogen, systemisch oder kontextuell. Gewalt verweist auf gerichtete Prozesse und auf Bruchstellen mit kontingenter Fortsetzung. Sie ist linear oder verschwommen, treibt den sozialen Wandel an oder behindert ihn; Gewalt ist (dys)funktional, sie enthält Rationalitäten, sie ist (nicht)utilitaristisch. Gewalt kann sich kollektiv und individuell zugleich organisieren, sie ist geschlechterbezogen, mit verantwortlichen Akteuren verbunden oder anonym. Einige Forscher gehen davon aus, dass ein Schlüssel zum Phänomen der Gewalt in einer ,dichten Beschreibung“ (Clifford Geertz) des Problems selbst liegt (Trotha 1999, 58-60). Inhärent enthält diese Forderung die Erwartung, über dichte Beschreibung ein empathisches Verständnis des Handelns von Akteuren zu schaffen, wie auch eine distanzierte Betrachtung der „objektiven Logik“ von 
Gewalt (Trotha a.a.O., 60). Diese von einem Soziologen in den ethnologischen Diskurs eingebrachte Forderung enthält Erwartungen an eine ethnologische Methodik, die Feldforschungsdaten mit vielschichtig interpretierten Folien der Deutung von Verhalten und symbolischen Zusammenhängen verbindet.

Die Verbindung von empirischer Feldforschung und Gewaltbeobachtung stellt in der Tat eine große Stärke ethnologischer Forschung dar. Doch trotz der Vielschichtigkeit des ethnologischen Zugangs können die daraus abgeleiteten Folien nur unzureichend zu einer gemeinsamen Beschreibung verdichtet werden, da sie zu viele epistemologische Widersprüche enthalten. Das Hauptproblem der Ethnologie besteht darin, inwiefern Täter und Opfern von Gewalthandeln Verständnis entgegengebracht werden sollte und wie mit dem damit verbundenen kulturellen Relativismus umzugehen ist. Dahinter steht, trotz der vielfältigen Ansätze ethnologischer Gewaltforschung, die Frage, wer unter welchen Umständen definiert, was als Gewalt zu verstehen ist. Neben Tätern und Opfern betreten hier auch die Zeugen das Forschungsfeld. Das Täter-Opfer-Zeuge-Dreieck wurde Mitte der 1980er Jahre durch David Riches in die Diskussion gebracht, dessen „Anthropology of Violence" (1986) im engeren Sinne als Geburtsstunde ethnologischer Gewaltforschung oder des systematischen ethnologischen Blicks auf die „dark side of human life“ (Schlee 2002 , 4) gilt. Mit Riches Herangehensweise ist hingegen auch ein methodischer Individualismus und Subjektivismus in der Gewaltanalyse verbunden, der nicht von allen Richtungen geteilt wird.

Ausgehend von einem in Großbritannien vorherrschenden semantischen Verständnis von „violence“, das eine Unterscheidung von legitimen und illegitimen Verhalten in der Anwendung von physischer Gewalt enthält, hat David Riches die Frage gestellt, nach welchen Kriterien in unterschiedlichen Kontexten über Legitimität/Illegitimität entschieden wird (Riches a.a.O, 1). Die von Riches eingeführte Methodik gibt einen Hinweis darauf, warum die Uneinigkeit darüber, was unter "Gewalt" verstanden werden kann, sowohl objektive als auch subjektive Gründe enthält. Mit der beschreibenden und analytischen Versprachlichung von „Gewalt" sind epistemologische Probleme verbunden. Ein Problembereich ist semantischer Art. Das deutsche Wort „Gewalt“ wird umgangssprachlich und wissenschaftshistorisch mit „Herrschaft“ und „Macht“ assoziiert (Elwert/Feuchtwang/Neubert 1999, 16). Der englische Begriff „,violence“ impliziert eine Trennung von „Legitimität“ und „Illegitimität“. Das französische Wort ,violence“ enthält darüber hinaus den Aspekt der Leidenschaft. Diese semantische Problematik verstärkt sich in den multilinguistischen Milieus, in denen Ethnologen meist arbeiten. Da zum ethnologischen Selbstverständnis die Absicht gehört, gesellschaftliche und kulturelle Prozess aus sich selbst heraus (mit dem Wissenschaftskürzel „emisch“ versehen) zu verstehen, treten hier permanent Zwänge der Übersetzungsleistung auf. Lassen sich Phänomene und Akteure wie gewaltsame Selbsthilfe, Krieg, Fehde, Razzia, Genozid, bewaffneter Jihad, Kopfjagd, prügelnde Ehemänner und Väter, Hooligans und Expertinnen der Frauenbeschneidung unter einem Nenner fassen? Sind gewaltregulierende und transportierende Institutionen und Praktiken wie Verfahren, Meidung, Hexerei, Ritual, Erinnerung und Vergessen, traumatische Störungen, Rache und Versöhnung, gewalttätige Sport- und Spielarten vergleichbar? Zentriert sich Gewalt auf den Körper? Was verstehen andere Gesellschaften unter Gewalt?

Gewiss gibt es einige grundsätzliche Gemeinsamkeiten ethnologischer Gewaltforschung. Gewalt wird in der Regel als sozio-kulturelle Kategorie betrachtet, die von Aggression als biologisch-physiologische Triebkraft unterschieden werden muss. Keine Einigung besteht indes in der Frage, welche Rückbindung sozio-kulturelle Verhaltensweisen und gewalttransportierende Symboliken an die biologische Natur des Menschen 
haben. Gewalt wird in der Regel nicht rein individuell oder per se als unsozial betrachtet, sondern ist sozialer oder kultureller Regelhaftigkeit ausgesetzt (Spencer 1993, 559). Weitgehende Einigkeit in der ethnologischen Gewaltforschung scheint offensichtlich auch bezüglich der Feststellung zu herrschen, dass ihr Beobachtungsfeld nicht ,jenseits des Staates" angesiedelt ist. Der Anspruch besteht darin, vergleichende Aussagen zu machen, die für alle Gesellschaftstypen und politischen Formationen und den damit verbundenen Akteuren - kollektiv oder individuell - gelten. Strukturelle Positionen in der ethnologischen Gewaltforschung, die von einer stufenweisen Vernestung häuslicher, lokaler, staatlicher (über Einschluss- und Ausschlusskategorien vermittelter) und politischökonomisch überdeterminierter Gewaltverhältnisse (Kapitalismus, Kolonialismus, Imperialismus, Neoliberalismus, Globalisierung) ausgehen, betrachten die Ausklammerung des Staates als wissenschaftlichen Sündenfall (Scheper-Hughes/Bourgois 2004). Aber auch Vertreter eines vergleichenden Blickes auf eine über den Krieg institutionalisierte Gewalt in tribalen Gesellschaften „ohne Zentralgewalt“ haben den Anspruch, dass Aussagen über Dynamiken der Aufrüstung und der Allianzformierungen auf das Feld internationaler Beziehungen übertragbar sind (Helbling 2006, 597). Zwischen beiden Richtungen besteht allerdings auch die erste schwerwiegende Bruchstelle im fachlichen Diskurs. Argumentiert die letztere Position im Sinne Hobbes, dass Staatlichkeit eine zivilisierende Auswirkung auf endemisches Kriegsverhalten unter Lokalgruppen in nicht-staatlichen Zusammenhängen hat (Helbling ebd.), betont die erstere Position die Kausalität zwischen der Ausdehnung von Staatlichkeit und Gewalt (Warren 2001, Scheper-Hughes/Bourgois a.a.O., 12) Ein Teil der Ethnologen, die sich mit dem Ende des Kalten Krieges von der vergleichenden Untersuchung kleiner Kriege in einfachen Gesellschaften der Analyse des Verhältnisses zwischen lokaler Gewalt und globalen Strukturen zuwandten, stützt diese Position. Gewalt und Krieg in der „tribalen Zone“ (Ferguson/Whitehead 1999) erscheinen hier als Ausläufer und Mimetik von Staatlichkeit. Nicht zuletzt erscheinen auch die in der älteren Literatur notorisch für ihre kriegerische Wildheit zitierten Yanomami-Indianer (Chagnon 1968) aus einer historischen Perspektive als Akteure oszillierender Gewalt unter randstaatlichen Bedingungen von langer Dauer (Elwert/Feuchtwang/Neubert 1999, 14).

Die möglicherweise innovativste Leistung ethnologischer Gewaltforschung liegt in der thematischen Übertragung von Gewaltphänomenen der Randstaatlichkeit, die räumlich am Rande von Staaten und Imperien gefasst werden können, auf Phänomene, die sich im Zuge der Entwicklungen nach dem Kalten Krieg in das Innere von Gesellschaften verlagern. Grundbedingungen für Randstaatlichkeit sind nun soziale Deregulierung, Staatszerfall, neue Formen globalisierter Ressourcenbewegungen, Arbeitsteilung- und Austauschbeziehungen, Widerstands- und Repressionsformen und die sich wandelnden Reproduktionsprozesse von Gewalt. Das Phänomen der Randstaatlichkeit kann auch mit anderen Begriffen gefasst werden, z.B. als ,gewaltoffener Raum“, als wirtschaftlich strukturierter "Gewaltmarkt" (Elwert 1997) oder einfach als Schattenreich (,shadows“) mit eigenen Regeln und - bezogen auf die Forschung - mit unsicherer Datenlage (Nordstrom 2004). In allen Fällen geht es um territoriale Fragmentierungen, im Rahmen derer organisierte Gewalt eine Symbiose mit rationaler Wirtschaftsführung bei der Ausbeutung von Rohstoffen und Menschen eingeht. Umstritten hingegen ist die Rolle von „Kultur“ in der Darstellung und Deutung von Gewalt. Ist Kultur eine legitimierende „Fassade“ vor dem Hintergrund systemischer Kanäle von Gewalt und Konflikt (Elwert 2002)? Gehört diese „Fassade“ zur bedeutungszuweisenden und performativ ausstrahlenden Substanz von Gewalt (Riches 1986; Krohn-Hansen 1994, 367) oder ist sie schmückendes Beiwerk? Die Krux der Darstellung liegt im Verhältnis zwischen strukturellen Zusammenhängen der 
Beobachtungsebenen, den Annahmen über systemische Stabilisierungen, Dynamiken und Blockaden von Gewalt- und Konfliktprozessen und kollektiven oder individuellen Motivationen und Handlungsoptionen im Gewalthandeln.

Auf der methodischen Ebene hat der willentliche oder erzwungene ethnographische Umgang mit Gewalt sich in Feldforschungserfahrungen eingeschrieben und zur vergleichenden Objektivierung von Felddaten und Analysen beigetragen (Nordstrom/Robben 1995; Köhler 1998; Sluka 2007, 207-221). Darüber hinaus hat in dieser Phase die Neuformierung globaler Feindbildzuschreibungen (Huntington etc.) entlang kultureller Grenzziehungen auch ethnologische Fragestellungen nach den Konstruktions- und Imaginationsbedingungen von Feindbildern, nach der Symbolisierung und dem Management von Hass, Emotion und Identität auf eine neue politische Ebene erhoben (Schlee 2002). Nicht zuletzt hat der Unwillen, sich einer Popularisierung kulturalistischer Feindbilder zu unterwerfen, das ethnologische Interesse an den konkreten Motivationsstrukturen von Gewalthandeln angespornt (Elwert 2001, 714).

Mit Blick auf andere Disziplinen, die sich den schattenhaften Gewaltzonen nähern (Soziologie, Politik, Kriminalistik), hat die Ethnologie sicher keinen Alleinstellungsanspruch auf das Potential dichter Beschreibung von Gewalt entwickelt. Das Fach verfügt jedoch über ein nützliches methodisches Arsenal der ethnographischen Beobachtungsformen, der holistischen Herangehensweise, des Vergleichens und des Umgangs mit dem vagen Informationsgehalt des „Hörensagens“ und der Gerüchte, die in latenten Gewaltsituationen oft an Stelle sicherer Informationen stehen (Simons 1995; Zitelmann 1998, 2002). Das Vorhaben, sich auch dem Schattenreich von al-Qaida ethnographisch zu nähern (Hauschild 2008, 32), wird auf einen Rückgriff auf das Hörensagen der untersuchten Akteure, auf deren Symbolik sowie auf einen systematischen Vergleich angewiesen sein.

\section{Hauptrichtungen der ethnologischen Gewaltforschung}

Es lassen sich gegenwärtig vier Hauptrichtungen der ethnologischen Gewaltforschung unterscheiden. 1) Die vergleichende Forschung diskutiert die Grundbedingungen für Krieg und Frieden anhand relativ übersichtlicher lokaler Verhältnisse in einfachen Gesellschaften oder anhand von historisch-archäologischen Beispielen. 2) Systemisch orientierte Ansätze verbinden die Gewalt unmittelbar mit einem Konflikt und fragen nach ihren Kanalisierungen, Einbettungen und Blockaden. 3) Kognitiv ausgerichtete Untersuchungen betonen die kulturellen und symbolischen Eigenarten des jeweiligen Gewalthandelns. 4) Die strukturelle Richtung untergliedert aktuelle Gewaltphänomene in lokale, intermediäre und globale Ebenen, verbindet lokal beobachtbare Phänomene von Gewalthandeln mit Einschluss- und Ausschlussmechanismen auf der intermediären Ebene und stellt Verknüpfungen zur politischen Ökonomie des jeweiligen gesellschaftlichen Zusammenhangs her.

Besonders die systemischen und die strukturellen Ansätze sind keine rein ethnologischen Domänen, sondern haben vielfältige Bezüge zur Soziologie, Politikwissenschaft und Ökonomie. Wenn dies im Verlauf der weiteren Darstellung nicht immer ausdrücklich betont wird, dann aus text-ökonomischen Gründen.

1. Krieg und Frieden in einfachen Gesellschaften. Systematisch-vergleichende Forschungen zu Krieg und Frieden in relativ unkomplexen Gesellschaften können genealogisch als eine Ahnenreihe der Gewaltforschung betrachtet werden. Diese Ahnenreihe führt zurück in die Hochphase des Kalten Krieges und der Angst vor dem Atomkrieg (Bräunlein 1995). Das Interesse an Krieg (und Frieden) in kleinen Gesellschaften beweg- 
te sich darüber hinaus im Schatten zweier Diskussionen: Einer der beiden Diskussionsstränge betrachtete das Verhältnis zwischen Krieg, Konflikt und biologischen Determinanten der Aggression, z.B. Napoleon Chagnons (1968) Forschungen zu den Yanomamo; der andere betraf die möglichen systemischen (ökologischen, demographischen, kulturellen) Beziehungen zwischen Krieg, Konflikt und Umwelt (Nettleship et al. 1975; Haas 1990; Bräunlein/Lauser 1995, IXff). Neben Feldforschungsdaten flossen hier auch umfangreiche, statistisch-vergleichende Literaturauswertungen ein. Gerade die partikularistische Tradition der durch Franz Boas in den USA institutionalisierten Cultural Anthropology hatte eine Vielzahl von Daten zu indianischen Gesellschaften gesammelt, die zeigten, wie auf relativ kleinem Raum sehr unterschiedliche Formen des Gewaltverhaltens und der Friedlichkeit existieren konnten. Diese Daten informierten Diskussionen über die biologischen und kulturellen Determinanten von Gewalt. Boas selbst förderte diese Debatten sehr früh durch die über ihn seit den 1920er Jahren initiierte Forschung von Margaret Mead und Ruth Benedict zu Sozialisation und Gewaltverhalten, deren Ergebnisse gegen die biologischen Annahmen der physischen Anthropologie gerichtet waren. In der Friedensanthropologie setzen sich diese Diskussionen heute fort (Howell/Willis 1989; Silverberg/Gray 1992, Bräunlein/Lauser ebd.). Die langjährige politische Rahmung der Forschung und die damit verbundene Möglichkeit, die Ethnologie als sozialen Kommentar für Gegenwartsfragen zu nutzen, schlägt sich nieder in Auseinandersetzungen zwischen Vertretern einer ausdrücklichen Friedensanthropologie und Strömungen, die von sozio-biologischen Grundvoraussetzungen des Gewaltverhaltens ausgehen (Sponsel/Gregor 1994, Marano 2000).

2. Systemische Bezüge. Systemische Gewaltforschung geht von einer Verbindung von Gewalt und Konflikt aus. Im Rahmen von Interessenkonflikten kann Gewalt ein instrumentelles Mittel der ,zielgerichtete(n) und ungewünschten Schädigung anderer“ (Elwert 2002, 336) sein, das vor allem als „Ausfalloption zielgerichteten Handelns“ (Elwert a.a.O., 330) zum Einsatz kommt, wenn andere Lösungsmöglichkeiten nicht greifen oder gewaltoffene Räume sich verselbständigt haben. Gewalt ist in jedem Gesellschaftstypus mehr oder weniger latent vorhanden. Genauso sind auch gewalthemmende Faktoren vorhanden. Gewalt kann der Normendurchsetzung wie der Schaffung neuer Normen dienen (Elwert a.a.O., 344). Außerdem muss „Gewalt im Kontext der gewaltkontrollierenden und gewaltperpetuierenden Institutionen analysiert werden“ (Elwert a.a.O., 362). Worauf es hierbei ankommt, ist der Grad der Einbettung in weitere institutionelle Zusammenhänge, die als Kanäle von Problemlösungen und Kontakten wirken können, die zur institutionalisierten Übertragung und Transformation von Gewalt, Konflikt und Interessengegensätzen beitragen. Die mögliche Spannweite von Entwicklungen reicht dann von Genozid und Zerstörung über Fehden, Krieg und Blutrache zu Lösungen über Verfahren und Meidung. Dabei können auch Friedensoptionen verschlossen, Situationsverbesserungen aus der internen Kommunikation ausgeklammert oder unverhandelbare Ziele gesetzt werden, bei denen nur die Kontinuität des Gewalthandelns im Zentrum steht (Eckert et al. 1999, 4f.). Meidungsverhalten selbst ist eine kritische Größe, denn Meidung steht auch für institutionellen Mangel, der im Konfliktfall rasch zu einer grenzenlosen Steigerung von Gewalt führen kann (Elwert 2004a, 30). Verfahrenslösungen, die zeitweilig eine Gleichheit von Kontrahenten herstellen, gelten hier als idealtypische Kanäle der Auflösung von Gewalt. „Kultur“ wird hier in erster Linie als legitimierende Fassade von Gewalthandeln verstanden, das durch materiellen Gewinn, Prestige oder Macht motiviert wird (Elwert 2002, 338). Motive können über längere und kürzere Zeiträume einem Wandel unterliegen. Sie können auch kontingentes und erratisches Gewalthandeln im Nachhinein „schmücken“ (Elwert a.a.O., 344). Motive verstärken bei den Akteuren stabile Muster 
von Handlungsketten im Gewalthandeln. Daraus leitet sich auch eine Warnung ab, nach „Gewaltursachen“ zu suchen (Elwert a.a.O., 362). Bei Motivlagen, die sich öffentlich gegen einen äußeren Gegner richten, besteht das engere Ziel oft in einer endostrategischen Mobilisierung, d.h. die Mobilisation bezieht sich auf ein Konkurrenzverhältnis im eigenen Lager (Eckert et al. 1999). Der systemisch verwendete Gewaltbegriff hat allerdings zahlreiche Gewaltfelder bewusst nicht im Blickfeld. Konsensuelle Gewalt, etwa körperlich schmerzliche Gewalt in Initiationsritualen, wie auch die Beschneidung von Frauen, fällt hier nicht unter den Gewaltbegriff. Auch abstrakte, symbolische oder strukturelle Gewalt wird ausgeklammert. Begründet wird dies mit einer hochgradig metaphorischen Übertragung und der damit assoziierten mangelnden „Trennschärfe“ zwischen sehr unterschiedlichen Phänomenen des Gewalthandelns (Elwert 2002, 337-338).

3. Kognitive Bezüge. Aus der kognitiven Perspektive ist Kultur keine Fassade des Gewalthandelns, sondern integraler Bestandteil der Konstruktion von Gewalt, der Zugang zur emischen Selbstsicht und individuellen Subjektivität der Akteure gibt (Schmidt/Schröder 2001, 1; Steward/Strathern 2002). „Gewaltpotentiale in den Köpfen“ (Ehre, Rache) sind dabei mindestens so wichtig wie strukturelle Konfliktursachen, z.B. materielle und statusbezogene Interessenkonflikte oder Umwelt- und Ressourcenkrisen (Orywal 1996, 34-35). Gewaltsame Konfliktlösungsstrategien werden in kulturellen Überzeugungssystemen als kognitive Schemata konzeptualisiert (Orywal a.a.O., 38). Während die systemische Herangehensweise in erster Linie kollektives Gewaltverhalten betrachtet, enthält die kognitive Gewaltforschung kollektive und individuelle Bezüge. Die kognitiven Strömungen sind theoriegeschichtlich in der kognitiven Anthropologie der 1960er und der symbolisch-interpretativen Ethnologie der 1970er Jahre zu verorten. Als Gründungstext einer ausdrücklichen Ethnologie der Gewalt mit stark kognitiven und symbolischen Bezügen gilt David Riches „Anthropology of Violence“ (1986). Einerseits isolierte Riches die ethnologische Gewaltforschung als eigenständiges Thema, andererseits entwickelte er eine individualistisch-transaktionalistische Perspektive, die das TäterOpfer-Zeuge-Dreieck in den Mittelpunkt des analytischen Interesses stellte. Damit wurde ein weites Tor für kognitive Perspektiven auf Gewalt geöffnet, die sich vor allem durch die Betonung von Subjektivität auszeichnen. Riches selbst hebt hervor, dass Gewalt eine Sache der individuellen Benennung und der Wahrnehmung von Performanz sei (Riches a.a.O., 6), wobei die Einschätzung der Legitimität/Nichtlegitimität von Gewalthandeln im Mittelpunkt steht. Riches hat aber auch auf der symbolischen Ebene kollektive Repräsentationen von Gewalt in Form von Mythologie und Ästhetik vor Augen. Gewalt kann auf diese Weise durch Symbole manifestiert werden, die metonymische und metaphorische Ausdrucksformen von Stärke vermitteln sollen (Riches a.a.O., 12). ${ }^{2}$ Der menschliche

2 Aus der empirischen Perspektive lässt sich darauf hinweisen, dass Gewalt und Stärke signalisierende Symbolik, wie unter den Palästinensern in den 1990er Jahren beobachtet, in keinem unmittelbaren Verhältnis zur Realpolitik stehen muss (Scheffler 1996). Ähnliche Beobachtungen konnte der Autor dieses Beitrages in den 1980er Jahren im Bereich der Oromo Liberation Front (OLF) in Äthiopien machen, wo eine archaische, Männlichkeit und Heldentum betonende Metaphorik über einen Verschriftlichungsprozess in eine politische Befreiungsrhetorik eingebaut wurde (Zitelmann 1991a). Der zum Beobachtungszeitraum relativ übersichtliche Rekrutierungsmechanismus von Kämpfern deutete eher darauf hin, dass die Guerilla an lokale Strategien, mit Hilfe derer junge Männer durch Gewalt Status erlangen konnten, anknüpfte. Der weitaus erfolgreichere Konkurrent auf diesem Gebiet war zu dieser Zeit (1984-1989) noch die äthiopische Armee, gegen die die OLF kämpfte. 
Körper als Ausgangspunkt und Gegenstand von schmerzhafter Gewalt spielt in dieser Symbolisierung eine große Rolle (Riches a.a.O., 11). Jene Perspektive auf die Bedeutung des körperlichen Schmerzes in der Symbolisierung von Gewalt wird von Aijmer (2000, 6) geteilt. Dieser unterteilt das Gewaltuniversum in drei Ebenen: 1) eine ethologischontologische Ebene, auf der die Empfindung physischer und psychischer Schmerzen verortet wird; 2) eine diskursiv unmittelbar zugängliche Ebene mit intentionalen Handlungen, in der Gewalt pragmatisch und im operationalen Sinne über soziale Organisation und Produktionsstrukturen wirken kann; 3) sowie eine Metaebene ikonischer Codes und imaginierter Ordnungen, die sich nur intuitiv und nicht sprachlich oder sensuell erschließen lässt (Aijmer a.a.O., 3-7). In der Verbindung der drei Ebenen kann Gewalt eindeutig dominieren (vermittelt durch Schmerz). Im offenen sozialen Diskurs kann Gewalt ein Zeichen mit vielfältiger Referenz sein und über die imaginären Codes kann Gewalt an psychisch verankerte Archaismen und implizites Wissen appellieren (Aijmer a.a.O., 7). In Grenzbereichen ist kognitive Gewaltforschung durch einen hochgradigen methodischen Individualismus gekennzeichnet. Einerseits wird das kreative Individuum in der Anwendung ,demokratischer Gewalt“ gefeiert, d.h. in der Bedeutung von Gewaltanwendung für sozial als „richtig“ eingeschätzte Veränderungen. Andererseits wird individuelle Kommunikationsverweigerung in transaktionalen Aushandlungsprozessen als „,nihilistische Gewalt" verdammt (Rapport 2000; Rapport/Overing 2007, 416-422). Forschungspragmatisch wichtig ist der aus der kognitiven Perspektive erfolgte Hinweis auf kollektive Gewaltdynamiken, die über vermutete magische Aggression (Zauberei, Hexerei, verantwortungszuschreibende Interpretationen von Krankheit und Unglück) oder Strategien der Wortgewalt (Klatsch, Gerüchte, alternatives Gerede über umstrittene Ereignisse) transportiert werden (Riches 1986, 8; Bryson 1992; Steward/ Strathern 2004).

4. Strukturelle Bezüge. Die strukturelle Gewaltforschung ist an Phänomenen der aktuellen Gewalt interessiert: Krieg (Revolution, Gegenrevolution), Staatsterrorismus und Folter, kommunale Gewalt, organisiertes Verbrechen und aggressive Kommodifizierungsprozesse (Menschen- und Organhandel, erzwungene Arbeitsmigration); an epistemischer Gewalt, die Einschluss und Ausschlussmechanismen schafft, die Gruppen marginalisiert und abwertet; an struktureller Gewalt, die einem System inhärent ist und über Armut, Hunger und Krankheit zum Ausdruck kommt (Warren 2001). Im Mittelpunkt stehen dabei drei miteinander verknüpfte Ebenen: 1) die lokale Ebene, das Stadtviertel, der Slum, die Haushalts- oder familiäre Ebene als unmittelbare Gewaltarenen; 2) die intermediären Arenen (urban, national) politischer Ordnungen, Hierarchien und Klassenstrukturen, über die Einschluss- und Ausschlussmechanismen erzeugt werden; 3) die übergeordneten politisch-ökonomischen Strukturen (Kapitalismus, Kolonialismus, Imperialismus, Neoliberalismus, Globalisierung). Strukturelle Gewaltforschung enthält das Selbstbild einer epistemischen Wende in der ethnologischen Gewalt- und Konfliktforschung, die zwischen „früher“ und ,heute“ scheidet. „Früher“ lag der Schwerpunkt funktionalistischer Forschung (der Social Anthropology britischer Prägung) in der Untersuchung interner Regulierungsmechanismen von sozialer Spannung basierend auf Machtpositionen und Gruppenfeindschaften in segmentären und lokalen Zusammenhängen. „Heute" betrachtet man den Zusammenhang zwischen lokalen Gemeinschaften, dem Staat und Globalisierungskonsequenzen (Warren a.a.O., 16202). Im Unterschied zu anderen Ansätzen ethnologischer Gewaltforschung ist die strukturelle Gewaltforschung sehr selbstbewusst in den Befreiungstheorien der 1960er Jahre (Jean-Paul Sartre, Frantz Fanon) und in der post-kolonialen Theorie verankert. Genozidalen Gewaltakten kommt in der strukturellen Gewaltforschung eine besondere Bedeutung zu. Dabei entwickelte sich eine generalisierte analytische Perspektive, die genozidale Gewalt in ein Gewaltkonti- 
nuum mit unspektakulären oder vermittelten Formen des Gewalthandelns einordnet, das mit Statuszuweisung, Ausschluss, semantischer oder praktischer Enthumanisierung und Entpersonalisierung, Pseudospezifizierung oder Verdinglichung in Form ethnischer Zuschreibungen verbunden ist (Scheper-Hughes/Bourgois 2004, 20-21). Der theoretische Schlüssel für diesen Zugang ist Pierre Bourdieus Ansatz der symbolischen Gewalt. Bourdieu hat damit im kabylischen Kontext Interaktionen zwischen Grundbesitzern und Pächtern beschrieben, die formal auf der Grundlage von Gleichheit und Reziprozität blieben, inhaltlich aber von Seiten der Grundbesitzer durch Strategien der Dominanz, die mit Missverständnissen über Absichten und Zusammenhänge operierten und dadurch Legitimität schufen, verbunden waren (Scheper-Hughes/Bourgois a.a.O. 21-23, Bourdieu 1976, 367-373). Der Weg in die Gewalthölle ist in der strukturellen Gewaltforschung mit strategisch gesetzten Missverständnissen gepflastert. Beschreibend knüpft diese Forschung an den radikalen, biographisch aufgebauten ethnographischen Realismus an, mit dem Oscar Lewis seine Darstellung der „Kultur der Armut“ illustrierte (Lewis 1966). Das Modell strukturell verkoppelter Ebenen von Gewalthandeln dient auch dazu, der einseitigen Ableitung von Armutssyndromen aus dem verantwortungslosen Verhalten der Armen und der damit verbundenen ideologischen Vereinnahmung, der Oscar Lewis mit seinem ethnographischen Realismus erlag, zu entgehen (Bourgois 2001).

In der ethnologischen Geschlechterforschung findet die strukturelle Gewaltforschung eine Ergänzung. In der Koppelung beider Fragestellungen geht es um die Bedeutung von körperlicher und psychischer Gewalt in sexuellen Beziehungen, um Gewalt gegen Frauen und um die Rolle der Gewalt in der Strukturierung von gender-Differenzen. Probleme wie die Beschneidung von Frauen oder häusliche Gewalt werden dabei nicht außerhalb einer generalisierten Gewaltforschung angesiedelt. Es geht vielmehr darum, die Deutungsmuster von Akteuren, die Gewalt gegenüber Frauen ausüben, mit von außen festgelegten Normen ins Verhältnis zu setzen (Ott 1998; Merry 2008, 20). Die Tendenz geht hier deutlich in die Richtung, transnationale Normensetzungen bezüglich der Stärkung von Frauenrechten dem empathischen Verständnis für Täterverhalten, Rituale und Bräuche vorzuziehen (Merry a.a.O., 2; Ströbele-Gregor 2004).

Gewalt und Natur. Das Verhältnis zwischen ethnologischer und (sozio)biologischer/ethnologischer Gewaltforschung stellt eine Besonderheit dar. Die Perspektivenkonkurrenz von Verhaltensforschung, (Sozio)Biologie und Ethnologie ist kontinuierlich (Elwert 2004b). Die einzelnen Forschungsrichtungen der Gewaltforschung nehmen dies unterschiedlich auf. In der klassischen, vergleichenden Forschung zu Krieg und Frieden ist das Thema dauerhaft präsent, auch weil in den USA die physische Anthropologie neben der Cultural Anthropology, der Linguistik und der Archäologie zum Kanon der ,vier Felder“ gehört, die unter dem Dach eines gemeinsamen fachlichen Zusammenhanges an Universitätsinstituten vertreten werden. In der strukturellen Gewaltforschung hingegen hat die Rückbindung von Gewaltverhalten an eine „Natur“ des Menschen keine Bedeutung. Kognitive und systemische Forschungen leiten aus der „Natur“ unter Verwendung ähnlicher Argumente unterschiedliche Perspektiven ab. Im kognitiven Ansatz werden kognitive Schemata aus natürlichen Grundbedürfnissen abgeleitet und in ideelle Bedürfnisse transformiert, die dann als handlungsleitende, ideelle Motivationen von Gewalt neben Macht, Prestige und materiellen Interessen stehen können (Orywal 2002, 121-124). Ähnlich begründet Aijmer das ethnologische Verständnis von Gewaltund körperlicher Schmerzempfindung als Ausgangspunkt für die Symbolisierung von Gewalt und Schmerz (Aijmer 2000, 4). Umgekehrt verweist auch Elwert im systemischen Argument darauf hin, dass sich seine Vorstellung von zielgerichteter und instrumenteller Gewalt aus physiologischen Überlegungen ableitet. Der Mensch ist ein soziales und em- 
pathiefähiges Wesen, das sich mit Hilfe von Institutionen eine sekundäre Umwelt schafft, über die Wahrnehmungen und Emotionen gesteuert werden. Hier unterscheidet sich Elwert nicht von kognitiven Positionen. Gewalt kann Risikowahrnehmungen reduzieren, sie kann mit Kooperation zusammen existieren, sie kann regelgebunden und regelbrechend sein. Es kann unterschiedliche soziokulturelle Gewaltordnungen mit unterschiedlichen Austragungsformen und Kanalisierungsmöglichkeiten von Gewalt geben (Elwert 2004b, 468). Im Mittelpunkt dessen steht die Annahme, dass mittels Gewalt die Risikowahrnehmung reduziert werden kann, und zwar durch kognitive Verengungen, Konzentrationen der Wahrnehmung sowie Signalisierung (Schreie, Blicke) auf eine Zielgröße. Aus diesem mit Gewalt verbundenen physiologischen Konzentrationszusammenhang leitet Elwert seinen sehr engen Blick auf den instrumentalen, strategischen und interessengebundenen Charakter von Gewalt ab (Elwert 1998, 3). Im Feldforschungskontext, der auf interpersonalen Kontakten, Situationswahrnehmungen und unmittelbarer Beobachtung beruht, gibt es im Prinzip keinen Raum, das Verhältnis von Gewalt und Natur empirisch zu überprüfen. Empirische Daten der ethnologischen Gewaltforschung leiten sich daher in der Regel aus dem allgemeinen theoretisch-methodischen Fundus der Ethnologie ab.

Die epistemologischen Herangehensweisen an Gewalt haben unterschiedliche Wurzeln und Schwerpunkte, aber sie überschneiden sich auch in den einzelnen Forschungstendenzen. Deutlich politisch engagiert ist die Positionierung der strukturellen Ausrichtung, die in den Traditionen von Befreiungsideologien der 1960er Jahre und den darauf folgenden post-kolonialen Diskussionssträngen - einschließlich der feministischen Anthropologie - steht (Scheper-Hughes/Bourgois 2004). Teile der systemischen Diskussion haben, neben der Soziologie Luhmanns, Wurzeln in den strukturalistisch-marxistischen Modellen von Produktionsweisen aus den 1970er Jahren, die nun aber stärker akteursorientiert umgedeutet wurden (Zitelmann 2004, 49-52). Es ist wenig bekannt, dass Claude Meillassoux einer der ersten war, der den Zerfall kleinbäuerlicher, hausgemeinschaftlicher Produktionsverhältnisse und die Reproduktionsbedingungen von Gewaltakteuren (revolutionär und gegen-revolutionär) unter der Bedingung chronischen Bürgerkrieges analysierte und damit Vorlagen für die systemische Herangehensweise an Gewaltmärkte gab (Meillassoux 1990). Bis in die 1980er Jahre war ethnologische Gewaltforschung in erster Linie an kollektiver Gewalt interessiert. Das Erbe des methodischen Kollektivismus durkheimscher Prägung blieb hier deutlich sichtbar. Das durch David Riches vertretene Täter-Opfer-Zeuge-Dreieck wurde zu einem Transportmittel für den methodischen Individualismus, der heute in allen Richtungen vorhanden ist. Auch strukturelle Gewaltforschung stellt die Frage nach dem kulturellen Kontext von Gewalt und fordert deren dichte Beschreibung, zeigt aber deutlich mehr Empathie für Opfer als für Täter (ScheperHughes/Bourgois a.a.O., 16, 26).

\section{Erinnerungen und Modelle}

Die ethnologische Gewaltforschung ist nicht von generellen sozialwissenschaftlichen Trends in der Betrachtung und Analyse von Gewalt zu trennen. Daran soll im folgenden Abschnitt ausdrücklich erinnert werden.

Die Institutionalisierung der Ethnologie als wissenschaftliches Fach in der zweiten Hälfte des 19. Jahrhunderts fand im Schatten kolonialer Eroberungen, beweglicher Grenzen sowie nationalistischer und industrieller Gewaltkonflikte statt. All dies ist auch in ethnologische Gewaltforschungen eingeflossen, die gleichzeitig über ein vielfältig vernetztes Theorieerbe mit der Soziologie und der Politikwissenschaft verbunden waren. Die 
Klassiker (Karl Marx, Gabriel Tarde, Emile Durkheim, Max Weber) diskutierten die Transformationen von Gewalt auf dem Weg in die bürgerlich-kapitalistische Gesellschaft und darüber hinaus. Klassenkampf (Marx) kanalisiert Gewalt und scheidet diese in nützliche, die Gesellschaft antreibende und in willkürliche Gewalt; Gesellschaft entwickelt sich über eine binär gedachte „universelle Opposition“, analog zu mechanischen und physischen Systemen und Körpern von Kraft und Gegenkraft (Tarde); Arbeitsteilung und Tausch (Durkheim) transformieren Gewaltverhältnisse in Solidarität und werden zur zweiten Natur der Menschen; Gewalt und Kampf werden zu demagogischer Rede, zu Wettbewerb, Konkurrenz und Wahl(kampf) veredelt (Weber). Frühe Sozialdarwinisten diskutierten die Transformation animalischer Überlebensinstinkte in menschliche Fähigkeiten der Jagd, der Kriegsführung und der entsprechenden Institutionalisierungen und Instrumentalisierungen (Spencer). Eine andere Version der Kontinuität animalischen und menschlichen Überlebenskampfes betont die Universalität ethnozentrischer WirGruppenbildungen unter den Bedingungen gewalttätiger Abgrenzungen und Feindbildschaffung (Sumner). Kontinentaleuropäische Varianten des Sozialdarwinismus vertraten die Eroberungstheorie des Staates (Nomaden überlagern Bauern) und waren in ihrer Theoriebildung hochgradig ethnologisch informiert (Gustav Ratzenhofer, Ludwig Gumplowicz, Franz Oppenheimer, Richard Thurnwald). Etwas später wurde dieser Ansatz in Verbindung mit der Idee des Klassenkampfes in aktivistische Elitetheorien mit faschistischer Grundtendenz transformiert (Georges Sorel, Vilfredo Pareto, Robert Michels). Doch nicht nur hier fanden Gewalttheorien eine semantische und thematische Transformation: Auch in Georg Simmels „Der Streit“ schwingt eine gewaltzentrierende Streitaxt mit. Simmels englische Übertragung „On Conflict“, die in der Soziologie viel stärker aufgenommen wurde als die deutsche Fassung, lässt semantisch offen, ob Interessengegensätze gewaltsam oder friedlich ausgetragen werden (Zitelmann 2004, 40-41).

In der Durkheimschen Soziologie, die selbst hochgradig ethnographisch informiert war, wurden die sozialdarwinistischen Motive des Kampfes und der Eroberung durch Motive der Arbeitsteilung und des friedlichen Austausches, d.h. durch Determinanten gesellschaftlicher Struktur und Entwicklung ersetzt (Durkheim 1988, 252-253). Unter dem Eindruck des 1. Weltkrieges und neuen ethnographischen Materials zur Allgegenwärtigkeit von Gabentausch und Gegenseitigkeit wertete Marcel Mauss den Tausch - sei es als Gabe oder über den Markt vermittelt - als Alternative gegenüber Gewalt und Krieg auf (Mauss 1978, 141f.). Über den Tausch erhielt seiner Ansicht nach auch die „Friedlichkeit" eine strukturierende Dimension, die mehr als nur die Abwesenheit von Gewalt und Krieg war.

In der Ethnologie lösten gegen Ende des 19. Jahrhunderts diffusionistische Überlegungen, die Migrationen von Bevölkerungen und Austausch kultureller Ideen, Praktiken und Artefakte in den Mittelpunkt des Interesses stellten, den Evolutionismus ab. In beiden Denkschulen fanden Vergleiche des Waffenarsenals der Völker statt. Entwickelten sich Waffentypen linear, von einfachen zu komplexen Formen? In welchem Verhältnis stand dies zu Jagd und Krieg? Kannten alle Völker Kriegswaffen? Was sagen die materiellen Formen der Waffen über die weiteren Kontakte ihrer Benutzer aus (Ratzel 1967). Der durch den frühen deutschen Diffusionismus geprägte Franz Boas wandte derartige Fragestellungen nach seiner Auswanderung in die USA auf differenzierte Betrachtungen von Gewalt und Friedfertigkeit an, die für die sich formierende US-amerikanische Cultural Anthropology zunächst erkenntnisleitend wurden. Boas hatte durch seine eigene frühe Feldforschung bei den Eskimo (1883-84) einen Gesellschaftstypus erfahren, in dem individuelle Gewaltakte zwar verbreitet waren, aber organisierte Gewalt in Form von Krieg und Fehden sehr selten vorkam (Boas 1964, 57, 70). 
In seinen weiteren Forschungen bei den Kwakiutl-Indianern an der amerikanischen Nordwest-Küste war er mit einem Gesellschaftstypus konfrontiert, der hierarchisch organisiert war und dessen dominierende Mitglieder ihre Ränge im Rahmen aggressiver Tauschwettbewerbe, dem Potlatch, aushandelten. Spätere ausführliche historische Arbeiten zum Potlatch-System zeigten eine regionale Transformation von chronischen Kriegsakten in unterschiedliche Stadien des Tauschwettbewerbs (Codere 1950). Implizit hatten diese Ähnlichkeiten mit Webers grundbegrifflichen Überlegungen zu graduellen Übergängen zwischen Gewalt, Krieg, Konkurrenz, Wettbewerb und Wahlkampf (Weber 1972, 20-22) im Gegensatz zu biologischen Determinierungen kriegerischen, gewalttätigen und abweichenden Verhaltens.

Nach dem 1. Weltkrieg wurde in den USA die Frage nach den Gründen für „abweichendes Verhalten“ im Schmelztiegel von neuen Immigranten, alteingesessenen „AngloSaxons" und den Nachkommen ehemaliger schwarzer Sklaven aufgeworfen. Wieder konkurrierten biologische und kulturelle Perspektiven auf das Phänomen der Gewalt (Bräunlein 1995, 15ff). Das relativ enge Nebeneinander von kriegerischen, wettbewerbsorientierten und relativ friedlichen Gesellschaften im nordamerikanischen Kontext informierte die frühe Cultural Anthropology, dass Gewalthandeln keine Sache der biologisch abgeleiteten Aggressivität war, die von manchen „Rassen“ eher als von anderen vertreten wurde (wovon die mit Boas konkurrierenden physischen Anthropologen ausgingen), sondern eine Sache der Kultur und der Sozialisation. Den 2. Weltkrieg begleiteten Ethnologen im Wesentlichen mit zwei zentralen Annahmen: In der amerikanischen Cultural Anthropology (Mead, Benedict) und in der britischen Social Anthropology (Malinowski) wurde die Perspektive vertreten, dass der Krieg eine späte Erscheinung im kulturellen und sozialen Leben der Menschheit sei. Demgegenüber betonte in Deutschland Wilhelm Mühlmann, in Form einer ethno-soziologisch argumentierende Unterstützung des NSRegimes, die grundsätzliche Bedeutung des Krieges für die Stabilisierung der sumnerschen Wir-Gruppe als ethnozentrischen Normalzustand und Urgrund des Politischen (Mühlmann 1940).

Mühlmanns Thesen unterschieden sich sachlich kaum von den Ergebnissen, die Social Anthropologists aus dem sub-saharischen Afrika mitbrachten und waren letztlich auch durch diese angeregt worden. Eine politische Ordnung in staatenlosen Gesellschaften die man über genealogisch gestaffelte patrilineare Abstammungsgruppen organisiert sah gestaltet sich demzufolge entlang der Segmente, die im Kriegsfall nach außen als kollektive Gewaltakteure mobilisierbar waren und im Friedensfall über interne Instanzen der Rechtsfindung verfügten (Radcliffe-Brown 1940, Evans-Pritchard 1940). Dies war jedoch nicht die einzige Botschaft, die die frühen Social Anthropologists in das Repertoire der ethnologischen Gewaltforschung aus Afrika einbrachten. Gewalt konnte auch indirekt auftreten, vermittelt durch Hexerei und Magie, wie bei den Azande (Evans-Pritchard 1937). Jeder Unglücksakt, der Individuen oder Gruppen betraf, jede soziale Missstimmung, die das Leben sozialer Gruppen über längere Zeit bedrückte, konnte mit magischer Gewalt böswilliger Menschen, Ahnen oder Geisterwesen verbunden werden. Gewalt wurde hier aus der unmittelbaren intentionalen und physischen Manifestation gelöst und mit Blick auf die Zuschreibung von Verantwortung interpretationsoffen behandelt. Der sich daraufhin in der Social Anthropology etablierende Blick auf gesellschaftliche Grundkonflikte zwischen Geschlechtern und Generationen sowie in der Organisation von Nachfolge und Erbrecht reduziert die Ursachen von Gewalt auf interne Konflikte (Gluckman 1955, Turner 1957). Auf der zeitlichen Ebene bewegt man sich hier im Rahmen der in der strukturellen Gewaltforschung als ,älter“ klassifizierten Herangehensweise an Gewaltphänomene. Sowohl Max Gluckman als auch Victor Turner waren sich dar- 
über klar, dass ihre analytischen Herangehensweisen nicht bloß lokale Dimensionen hatten, sondern sie rückten auch das Migrationsverhalten sowie die Urbanisierungshintergründe und Neuformierungen kollektiver politischer Akteure (z.B. Gewerkschaften in Südafrika) ins Blickfeld. Vor diesem Hintergrund gibt es für eine Anthropologie der Gewalt keinerlei Anlass, auf diese älteren analytischen Dimensionen zu verzichten.

Phänomenologische Ansätze der ethnologischen Gewaltforschung gehen heute oft von einem methodischen Individualismus mit sichtbaren und verantwortlichen Akteuren aus. Demgegenüber steht die ethnographische Beobachtung von Langzeitprozessen, der zufolge Situationen und Phasen der sichtbaren Gewalt und der relativen Friedlichkeit (in Slums, in Flüchtlingslagern, im Vorfeld von Bürgerkriegssituationen oder Genoziden, in Post-Gewalt-Konflikt-Situationen) fließend ineinander übergehen oder nebeneinander bestehen (Zitelmann 1991b; Malkii 1995; Chatterji/Mehta 2007; Das 2007). Gewalt scheint dann weniger instrumentell, utilitaristisch und linear zu sein, sondern eher kontingent, anonym und selbstorganisiert bzw. ,auto-poietisch“ (Feldman 1995). Indikatoren für Ängste über die Veralltäglichung von Gewalt sind oft Gerüchte. In ihnen werden in vager und interpretativ offener Form potentielle Täter, Opfer, Gewaltsituationen und Hintergrundzusammenhänge, z.B. Vermutungen über Verbindungen lokaler und globaler Zusammenhänge, kommuniziert (Taussig 1984; Zitelmann 1998). Bei näherer situativer Betrachtung löst sich die in Gerüchten anonym erscheinende Gewalt manchmal in transaktional aufgebaute Netze des Informationsaustausches (Simons 1994) oder der interessierten Desinformation auf. Auch lang hingezogene Veralltäglichung von Gewalt kann in Erfahrungsschritte von interessierten Akteuren zerlegt werden, nach denen instrumentelle Gewalt im Bruch von alten und in der Setzung neuer Normen mit mehr oder weniger Erfolg verbunden wird (Eckert 2003). Lokalisierte Gewaltakteure versuchen Legitimationsstrukturen über Allianzen mit globalen Partnern zu schaffen, so z.B. im Falle der Kommunikation von Terrorismusverdacht. In solchen Situationen, etwa während des somalischen Bürgerkriegs, zeigt sich im besonderen Maße die Ebenenverbindung zwischen dem klassischen lokalen Feld ethnologischer Gewaltbeobachtung in segmentären Gesellschaften und globalen Strukturen der Gewaltvermittlung und -kontrolle. Gewaltakteure formieren sich auf der Grundlage lokaler Gegebenheiten in Verbindung mit externen Chancen der Unterstützung. In den auf mündlicher Kommunikation beruhenden lokalen Strukturen verfließen Gerüchte mit jedem Allianzwechsel oder Friedensschluß. Im globalen Sicherheitsdiskurs können sie sich gleichzeitig zu handlungsanleitenden Nachrichten verhärten (Zitelmann 2003).

Besonders Beispiele aus Lateinamerika und Neuguinea informierten die vergleichende Forschung zu Krieg und Frieden in einfachen Gesellschaften. Gegeben ist in all diesen Gesellschaften ein Verhältnis zwischen kollektiver Gewalt in Form von Krieg und Fehden, der lokalen politischen Organisation und den Geschlechterbeziehungen. Die Artikulation dieser Verhältnisse und ihre interpretierende Deutung fallen sehr unterschiedlich aus. So ging Napoleon Chagnon (1968) davon aus, dass bei den südamerikanischen Yanomamo-Indianern die grundsätzlich aggressive Disposition des Menschen in Reinform erhalten geblieben sei. Kriege sind dann eine Form der Gewalt auf einer Stufenleiter von Aggressionsakten, die ihre dauernde Basis in Familien- und Geschlechterbeziehungen haben. Im Mittelpunkt der Gewaltanalyse steht ein aggressives Männlichkeitsideal, das mit Wildheit und Gewalttätigkeit assoziiert wird.

Helbling (1999; 2006, 599) analysiert eine völlig andere Dynamik. Hier sind es strukturelle Rahmenbedingungen (Demographie, Umwelt), die kriegerische Interaktionen zwar in Gang setzen, aber nicht die eskalierende Dynamik bestimmen. Unter den Bedingungen einer fehlenden übergeordneten Sanktionsinstanz, lokal konzentrierten Ressourcen und 
hohen Opportunitätskosten der Abwanderung in neue Siedlungsgebiete entwickeln sich die Handlungsmöglichkeiten von Gruppen spieltheoretisch nach dem Maßstab des Gefangenendilemmas. Friedensbereitschaft wird für jede Gruppe zu riskant, weil Nachbarn sie ausnutzen könnten. Zur Reduktion der Lebensunsicherheit wird eine Lokalgruppe ständig zur Fortsetzung einer kriegerischen Konfrontationsideologie gezwungen.

Eine wiederum andere Perspektive auf Kriege unter lateinamerikanischen Tieflandindianern hat der Strukturalist Pierre Clastre $(1976$; 1997) entwickelt. Dieser feiert die lange Staatsresistenz lateinamerikanischer Tieflandindianer als Errungenschaft. Clastre versteht die endemischem Kleinkriege, das „warring“ (bei Hobbes „warre“) unter benachbarten Kleingruppen als Instrument gegen den Staat. Anders als Helbling betont Clastre keine spieltheoretisch begründeten Zwänge, sondern eine voluntaristische Abneigung gegen Hegemonie. Keine Gruppe kann auf diese Weise auf Dauer Macht über andere erlangen. Oszillierende Gewalt schafft die notwendige territoriale Distanz zwischen Gruppen. Der gefährliche Zwischenraum - die reine Natur - diente der Jagd, der Abwehr von Epidemien, dem Ritual und als räumliche Fläche für Allianzen und Frauentausch. Clastre steht für den gewagten Versuch, die klassischen Schnittstellen von Kultur und Natur in der strukturalistischen Theorie levi-strausscher Prägung (Frauentausch, Mythos) um den Faktor der Gewalt zu ergänzen.

Helbling und Clastre gehen in ihren Modellen davon aus, dass sich Lokalgruppen in Gewaltzusammenhängen relativ mechanisch verhalten. Die kollektive Existenz der lokalen Gruppen wird als gegeben vorausgesetzt. Dem widerspricht ein von Harrison (1989) vorgestelltes Beispiel aus Neuguinea. In Dörfern organisierte Lokalgruppen erscheinen hier als grundsätzlich fragile Einheiten, deren Bewohner über zahlreiche äußere Verwandtschafts- und Klanbeziehungen verfügen und bei denen die Tendenz zu einem fluiden Wechsel zwischen Siedlungsgebieten groß ist. Das Zusammenleben von Menschen wird im Allgemeinen als ein Zustand der zwischenmenschlichen Gewaltlosigkeit und des Friedens idealisiert. Dieser Frieden erlaubt jedoch auch eine relativ hohe Mobilität, die auf Kosten der Lokalgruppe und des Dorfes geht. Das politische Ziel, das Dorf zu erhalten und die äußeren Beziehungen zu kappen, setzt eine Gewaltsituation voraus. Diese Gewaltbereitschaft müssen die Männer erst durch ritualisiertes männliches Gemeinschaftsverhalten in Form von organisierten Männerbünden erlernen. Gewaltbereitschaft wird in der sekundären männlichen Sozialisation trainiert. „Natürlich“ ist der Frieden. Ist das „Dorf“ als Einheit konsolidiert, tritt der friedliche Zustand auch wieder ein.

Die unterschiedlichen faktischen und interpretativen Möglichkeiten im Umgang mit lokaler kollektiver Gewalt lassen sich nach Belieben ergänzen. Es gibt keine allgemeinen Regeln, bestenfalls regionale und zeitliche Trends. Aus der Vielgestaltigkeit des Untersuchungsgegenstandes lässt sich begründen, dass analytische Zielgenauigkeit nur durch selektive Begrenzung auf bestimmte Gewaltformen erreicht werden kann.

\section{Kulturrelativismus und Gewalt}

Mein Prolog sollte den Leser in das durch eine bedrohliche Natur umgebene Gewaltreich des Mr. Kurtz führen und dabei Zusammenhänge mit der ethnologischen Gewaltforschung suggerieren. Gewaltaktionen im lokalen Kontext und in einem über unterschiedliche Ebenen vermittelten Kontext zielen beide auf die Herstellung eines „Naturzustandes“. Die einen erreichen diesen durch abschreckende Wildheit oder den distanzierenden „,natürlichen" Zwischenraum. Für die anderen können inszenierte Plötzlichkeit und Anonymität von Gewalt und Terror in urbanen Situationen einen symbolisch analogen Einbruch 
der „Natur“ in den Alltag ausdrücken, über die Unverantwortlichkeit und Angst transportiert werden (Elwert 1998). Joseph Conrad war ein Meister der erzählten Naturalisierung von Gewalt. Literatur ist Kunst und erschließt sich bekanntlich in den Augen der Betrachter. Konstruktivistische Forschungsperspektiven in der Ethnologie behaupten ähnliches im Hinblick auf Gewalt (Rapport/Overing 2007, 417) und die Wahrnehmung von Natur (Luig 2002). Am Ende folgt daraus die Problematik des Kulturrelativismus und des Subjektivismus in der Gewaltforschung.

Der Umgang mit dem Kulturrelativismus, d.h. die Frage, inwieweit man allgemeine Standards an das Gewaltverständnis herantragen kann oder die „emische“ Selbstsicht und kulturelle Übereterminierung der Akteure verstehen muss, bewegt seit langem die ethnologische Gewaltforschung (Heelas 1982; Krohn-Hansen 1995; Ferguson/Whitehead 1999; Gabbert 2004). Diese Problematik hat zunächst eine unmittelbare Rückkoppelung an die Datengewinnung aus der Feldforschung zur Folge. Jan Köhler, der Anfang der 1990er Jahre Gewaltdynamiken in Georgien beobachtete, hat die Diskussion über Denkgebote zur Gewalt um zwei ratgebende Zitate aus der Feldforschungssituation heraus erweitert:

„Du wirst viele Dinge in Deinem Leben treffen, die Du nicht liebst, aber das heißt nicht, dass es diese Dinge deshalb nicht gibt.“

„Warum beschäftigst Du Dich mit den Schattenseiten einer Gesellschaft, die es überall gibt. Beschäftige Dich lieber mit der georgischen Gastfreundschaft, die Dir zuteil wurde.“ (Köhler 1998, 18, 20)

In der einen oder anderen Weise müssen ethnologische Feldforscher/innen in Gewaltsituationen zwischen Gastfreundschaft und Kritik, Nähe und Distanz, Legitimität und Illegitimität und dem, was sie lieben oder nicht lieben, lavieren. Die Möglichkeiten und Grenzen des Verständnisses von Relativität werden dabei nicht nur durch die Kontakte zu Informanten bestimmt, sondern auch durch das theoretische und methodische Gepäck und den Zeitgeist.

Über die Ethnologie hinaus wurde hier die von Renato Rosaldo vorgetragene Selbstsicht des Kopfjägers populär, den die Trauer um den Tod eines Kindes in eine emotionale Rage bringt, aus der er sich nur durch Kopfjagd erlösen kann (Rosaldo 1993). Nun ist der vergleichende Blick auf Trauer, Schuldzuweisungen, Gemütsbewegungen und mehr oder weniger gewalttätige Lösungen und Transformationen gewalttätiger Prozesse nicht ganz neu, sondern gehört zum klassischen Repertoire der Cultural Anthropology (Benedict 1955, 87ff.). Der ältere Kulturrelativismus, vor dessen Hintergrund Benedict argumentierte, ging beschreibend vom Potential menschlicher Möglichkeiten aus. Der Kulturrelativismus, zu dem Rosaldo einen Beitrag liefert, diskutiert Kultur als verengte Determination von subjektiver Realität (Fernandez 2001, 3111). Diese Sichtweise auf das „Gefängnis Kultur" (Caglar 1990) ist sicher nicht unumstritten.

Als ständiger Subtext durchzieht die neuere ethnologische Gewaltforschung das Thema Holocaust und die Verantwortung für das nationalsozialistische Gewaltregime als eine Form der Organisation von Genozid, sowie die Vergleichbarkeit mit anderen Genoziden und deren Verantwortungszusammenhängen (Elwert/Feuchtwang/Neubert 1999, 30; Whitehead 2004, 4; Scheper-Hughes/Bourgois 2004, 19f.). Außerhalb des deutschsprachigen Bereichs ist wenig bekannt, mit welchem Fundus an sozialdarwinistischen, sozialund kulturanthropologischen Wissen der Ethnologe Wilhelm Mühlmann nach der Machtergreifung Hitlers für ein Verständnis der inneren Logik und des symbolischen Instrumentariums der nationalsozialistischen Bewegung warb (Mühlmann 1933). Elwert, der in der Gewaltforschung den Fassadencharakter von Kultur betont, war später kritischer 
Schüler Mühlmanns. Es bietet sich an, hier eine Wurzel der Kritik an kulturalisierenden und symbolischen Deutungen von Gewalt zu sehen.

Speziell die deutsche Ethnologie enthält noch andere Merkmale der Verbindung von Gewalt und Zeitgeist. In der kulturhistorischen Schule der älteren deutschen Ethnologie (Leo Frobenius, Adolf E. Jensen) wurde Kopfjagd mit dem „Töterkomplex“ (wahlweise auch Verdienst- oder Megalithkomplex) assoziiert. Dahinter stand die Vorstellung, dass sich von Europa, über Afrika bis nach Südostasien ein Kulturkreis spannte, der sich durch eine besondere Form ritualisierter Gewalt, Trophäenjagd, Heldenverehrung und Erinnerung tragende Grabbauten auszeichnete. Die Menschen in Agrar- und Jägergesellschaften litten darunter, dass sie die Gaben der Natur, die ihnen zum Leben dienten, in immer wiederholenden Zyklen „töten“ mussten. Adolf Jensen hat daraus nach dem 2. Weltkrieg das „religiöse Weltbild einer frühen Kultur" erschrieben, in dessen Mittelpunkt die geschlechterbezogene Mythologisierung von Tod und Zeugung stand (Jensen 1949). Männer „mussten“ töten, wie Frauen gebären mussten.

Abstrahiert man von den ethnographischen Daten und der vergleichenden Methodik, führt die Theoriebildung auf einer Schiene zurück in die Welt männlicher Teilnehmer am 1. Weltkrieg. Die ethnographische Durchsetzung des „Töterkomplexes“ unterstützte eine Selbstsicht, nach der sinnloses, desinteressiertes Töten ehrenhaftes Heldentum sei, wenn Brauch und Kult dies verlangten (Braukämper 2001, 232). So gesehen wäre der ethnologische „Töterkomplex“ ein kleinstes Teilchen im Aufbau und der Fassade der ideologischen Schlachtordnung und der Gewaltspirale, die Enzo Traverso als „Bann der Gewalt“ in einem langwierigen „europäische(n) Bürgerkrieg“ bezeichnet (Traverso 2008). Nur aus der Distanz wird man sehen können, in welchem Banne aktuelle ethnologische Gewaltwahrnehmungen stehen.

\section{Bibliographie}

Aijmer, G. (2000) Introduction: The Idiom of Violence in Imagery and Discourse. In: Aijmer, G./Abbink, J. (Ed.) Meanings of Violence. A Cross-Cultural Perspective. Oxford: Berg.

Benedict, R. (1955). Urformen der Kultur. Reinbeck: Rowohlt.

Boas, F. (1964) The Central Eskimo [1888]. Lincoln, NE: University of Nebraska Press.

Bourdieu, P. (1976) Entwurf einer Theorie der Praxis. Frankfurt a.M.: Suhrkamp.

Bourgois, P. (2001) Poverty, Culture of. In: Smelser, N. J./Baltes, P. (Ed.) International Encyclopedia of the Social and Behavioral Sciences (Vol. 17). Amsterdam/Paris/New York: Elsevier.

Braukämper, U. (2001) Der 'Verdienst-Komplex'. Rückblick auf einen Forschungsschwerpunkt der deutschen Ethnologie. In: Zeitschrift für Ethnologie, 126: 209-236.

Bräunlein, P. J./Lauser, A. (1995) Auf dem Weg zu einer Ethnologie des Krieges und des Friedens. In: Bräunlein, P. J./Lauser, A. (Ed.) Krieg und Frieden. Ethnologische Perspektiven. Bremen: kea-Edition.

Bräunlein, P. J. (1995) Ethnologie an der Heimatfront: zwischen Heilslehre, Kriegswissenschaft und Propaganda. Margaret Mead, die amerikanische cultural anthropology und der II. Weltkrieg. In: Bräunlein, P. J./Lauser, A. (Ed.) Krieg und Frieden. Ethnologische Perspektiven. Bremen: kea-Edition.

Bryson, K. (1992) Just Talk: Gossip, Meetings, and Power in a Papua New Guinea Village. Berkeley/Los Angeles/London: University of California Press. 
Caglar, A. S. (1990) The Prison House of Culture in the Study of Turks in Germany. Berlin: Das Arabische Buch (Sozialanthropologische Arbeitspapiere, 31).

Chagnon, N. A. (1968) Yanomamo: The Fierce People. New York: Holt, Rinehart \& Winston.

Chatterji, R./Mehta, D. (2007) Living with Violence. An Anthropology of Events and Everyday Life. London/New York/New Delhi: Routledge.

Clastres, P. (1976) Staatsfeinde. Studien zur politischen Anthropologie. Frankfurt: Suhrkamp.

Clastres, P. (1997) Archéologie de la Violence. La Guerre dans les sociétés primitives. La Tour d'Aigues: éditions de l'aubé.

Codere, H. (1950) Fighting with Property: A Study of Kwakiutl Potlatching and Warfare, 1792-1930 New York: J.J. Augustin.

Conrad, J. (1973) Heart of Darkness [1902]. Harmondsworth: Penguin Books.

Das, V. (2007) Life and Words: Violence and the Descent into the Ordinary. Berkeley: University of California Press.

Durkheim, E. (1988) Über soziale Arbeitsteilung. Frankfurt a.M.: Suhrkamp.

Eckert, J. M. (2003) The Charisma of Direct Action. Power, Politics, and the Shiv Sena. New Delhi/ Oxford/ New York: Oxford University Press.

Eckert, J. M./Elwert, G./Gosztonyi, K./Zitelmann, T. (1999) Konflikttreiber - Konfliktschlichter. Vergleichende Forschungen in Bosnien, Bombay und Oromiya Regional State (Äthiopien). Berlin: Das Arabische Buch (Sozialanthropologische Arbeitspapiere ,75).

Elwert, G./Feuchtwang, S./Neubert, D. (1999) The Dynamics of Collective Violence An Introduction. In: Elwert, G./Feuchtwang, A./Neubert, D. (Ed.) Dynamics of Violence. Processes of Escalation and De-Escalation in Violent Group Conflicts. Berlin: Duncker \& Humblot.

Elwert, G. (1997) Gewaltmärkte. Beobachtungen zur Zweckrationalität der Gewalt. In: Trotha, T. v. (Ed.) Soziologie der Gewalt. Opladen: Westdeutscher Verlag.

Elwert, G. (1998) Vorwort: Gewalt als inszenierte Plötzlichkeit. In: Koehler, J./Heyer, S. (Ed.) Anthropologie der Gewalt. Chancen und Grenzen der sozialwissenschaftlichen Forschung. Berlin: VWF Verlag für Wissenschaft und Forschung.

Elwert, G. (2001) Gewaltmärkte. In: Haug, W. F. (Ed.) Historisch-Kritisches Wörterbuch des Marxismus (Bd. 5). Hamburg: Argument-Verlag.

Elwert, G. (2002) Sozialanthropologisch erklärte Gewalt. In: Heitmeyer, W./Haggan, J. (Ed.) Internationales Handbuch der Gewaltforschung. Opladen: Westdeutscher Verlag.

Elwert, G. (2004a) Anthropologische Perspektiven auf Konflikt. In: Eckert, J. M. (Ed.) Anthropologie der Konflikte. Georg Elwerts konflikttheoretische Thesen in der Diskussion. Bielefeld: transcript Verlag.

Elwert, G. (2004b). Biologische und sozialanthropologische Ansätze in der Konkurrenz der Perspektiven. In: Heitmeyer, W./Soeffner, H.-G. (Ed.) Gewalt. Entwicklungen, Strukturen, Analyseprobleme. Frankfurt a. Main: Suhrkamp.

Evans-Pritchard, E. E. (1937) Witchcraft, Oracles and Magic among the Azande. Oxford: Oxford University Press.

Evans-Pritchard, E. E. (1940) The Nuer of the Southern Sudan. In: Fortes, M./EvansPritchard, E. E. (Ed.) African Political Systems. Oxford: Oxford University Press.

Feldman, A. (1995) Ethnographic States of Emergency In: Nordstrom, C./Robben, A. (Ed.) Fieldwork under Fire. Contemporary Studies of Violence and Survival. Berkeley/Los Angeles/ London: California University Press. 
Ferguson, B. R./Whitehead, N. L. (Ed.) (1999) War in the Tribal Zone. Expanding States and the Indigenous Warfare (2nd ed.). Oxford/ Santa Fe, NM: James Currey/School of American Research Press.

Ferguson, B. R./Whitehead, N. L. (1999) Preface to the Second Edition. In: Ferguson, B. R./Whitehead, N. L. (Ed.) War in the Tribal Zone. Expanding States and Indigenous Warfare. Oxford/ Santa Fe, NM: James Currey/School of American Research Press.

Fernandez, J. (2001) Cultural Relativism, Anthropology of. In: Smelser, N. J./Baltes P. (Ed.) International Encyclopedia of the Social and Behavioral Sciences (Vol. 49). Amsterdam: Elsevier.

Gabbert, W. (2004) Was ist Gewalt? Anmerkungen zur Bestimmung eines umstrittenen Begriffes. In: Eckert, J.M. (Ed.) Anthropologie der Konflikte. Georg Elwerts konflikttheoretische Thesen in der Diskussion. Bielefeld: transcript Verlag.

Gluckman, M. (1956) Custom and Conflict in Africa. Oxford: Oxford University Press.

Haas, J. (1990) The Anthropology of War. Cambridge: Cambridge University Press.

Harrison, S. (1989) The Symbolic Construction of Aggression and War in a Sepik River Society. In: Man, 24: 583-599.

Hauschild, T. (2008) Ritual und Gewalt. Ethnologische Studien aus europäischen und mediterranen Gesellschaften. Frankfurt a.M.: Suhrkamp.

Heelas, P. (1982) Anthropology, Violence and Catharsis. In: Marsh, P./Campbell, A. (Ed.) Aggression and Violence. Oxford: Basil Blackwell.

Helbling, J. (1999) The Dynamics of War and Alliance among the Yanomani. In: Elwert, G./Feuchtwang, S./Neubert, D. (Ed.) Dynamics of Violence. Processes of Escalation and De-Escalation in Violent Group Conflicts. Berlin: Dunker \& Humblot.

Helbling, J. (2006) Tribale Kriege. Konflikte in Gesellschaften ohne Zentralgewalt. Frankfurt/ New York: Campus Verlag.

Howell, S./Willis, R. (Ed.) (1989) Societies at Peace. Anthropological Perspectives. London: Routledge.

Jensen, A. E. (1949) Das religiöse Weltbild einer frühen Kultur. Stuttgart: August Schröder Verlag.

Koehler, J./Heyer, S. (1998) Einleitung: Soziologisches Sprechen und empirisches Erfassen - Explaining Violence. In: Koehler, J./Heyer, S. (Ed.) Anthropologie der Gewalt. Chancen und Grenzen der sozialwissenschaftlichen Forschung. Berlin: VWF Verlag für Wissenschaft und Forschung.

Krohn-Hansen, C. (1994) The Anthropology of Violent Interaction. In: Journal of Anthropological Research, 50 (4), 367-381.

Lewis, O. (1966) La Vida: A Puerto-Rico Family in the Culture of Poverty. New York/ London: Secker \& Warburg.

Luig, U. (2002). Einleitung. In: Luig, U./Schultz, H.-D. (Ed.) Natur in der Moderne. Interdisziplinäre Ansichten. Berlin: Geographisches Institut der Humboldt-Universität zu Berlin

Malkkii, L. (1995) Purity and Exile. Violence. Memory and National Cosmology among Hutu Refugees in Tanzania. Chicago, IL: University of Chicago Press.

Marano, L. (2000) Analysis: Darkness in Anthropology, United Press International, 21. Oct. 2000. http://www.nku.edu/ humed1/darkness_in_el_dorado/documents/0163.htm (23.02.2009)

Mauss, M. (1975) Die Gaben und die Verpflichtung sie zu erwidern. In: Mauss, M. Soziologie und Anthropologie, Bd. II . Frankfurt/ Berlin/ Wien: Ullstein.

Meillassoux, C. (1990) Poissons à brûler: du poisson dans l'eau à la terre brûlée. Paris: CNRS (Manuskript). 
Merry, S. E. (2008). Introduction. In: Merry, S. E. (Ed.) Gender Violence: A Cultural Perspective. Oxford: Wiley-Blackwell.

Mühlmann, W. E. (1933) Die Hitler-Bewegung. Bemerkungen zur Krise der bürgerlichen Kultur. Zeitschrift für Völkerpsychologie und Soziologie, 9: 129-139.

Mühlmann, W. E. (1940) Krieg und Frieden. Ein Leitfaden der Politischen Ethnologie. Heidelberg: Carl Winter's Universitätsbuchhandlung.

Nettleship, M. A./Dalegivens, R./Nettleship, A. (Ed.) (1975) War, Its Causes and Correlates. The Hague/Paris: Mouton Publishers.

Nordstrom, C. (2004) Shadows of War, Violence, Power, and International Profeetering in the Twenty-First Century. Berkeley/Los Angeles/London: University of California Press.

Nordstrom, C./Robben, A. (Ed.) (1995) Fieldwork under Fire. Contemporary Studies of Violence and Survival. Berkeley/Los Angeles/London: University of California Press.

Oryval, E. (1996) Krieg und Frieden in den Wissenschaften. In: Oryval, E./Rao, A./ Bollig, M. (Ed.) Krieg und Kampf. Die Gewalt in unseren Köpfen. Berlin: Reimer.

Oryval, E. (2002) Krieg oder Frieden. Eine vergleichende Untersuchung kulturspezifischer Ideale - Der Bürgerkrieg in Belutschistan/Pakistan. Berlin: Reimer.

Ott, L. (1998) Legitimität, Angst und Schmerzen. Zwei Beispiel über physische Alltagsgewalt gegen Frauen in Samburu, Kenia. In: Koehler, J./Heyer, S. (Ed.) Anthropologie der Gewalt. Chancen und Grenzen der sozialwissenschaftlichen Forschung. Berlin: VWF Verlag für Wissenschaft und Forschung.

Radcliffe-Brown, A. R. (1940) Preface. In: Fortes, M./Evans-Pritchard, E. E. (Ed.) African Political Systems. Oxford: Oxford University Press.

Rapport, N. (2000) 'Criminal by Instinct': On the 'Tragedy' of Social Structure and the 'Violence' of Individual Creativity. In: Aijmer, G./Abbink, J. (Ed.) Meanings of Violence. A Cross-Cultural Perspective. Oxford: Berg.

Rapport, N./Overing, J. (2007) Violence. In: Rapport, N./Overing, J. (Ed.) Social and Cultural Anthropology. The Key Concepts (2nd.ed.). London/ New York: Routledge.

Ratzel, F. (1967) Über den anthropogeographischen Wert ethnographischer Merkmale [1912]. In: Schmitz, C. A. (Ed.) Historische Völkerkunde. Frankfurt a. M.: Akademische Verlagsanstalt.

Riches, D. (Ed.) (1986) The Anthropology of Violence. Oxford: Blackwell.

Riches, D. (1986) The Phenomenon of Violence. In: Riches, D. (Ed.) The Anthropology of Violence. Oxford: Blackwell.

Rosaldo, R. (1993) Der Kummer und die Wut eines Kopfjägers. Über die kulturelle Intensität von Emotionen. In: Berg, E./Fuchs, M. (Ed.) Kultur, soziale Praxis, Text. Die Krise der ethnographischen Repräsentation. Frankfurt: Suhrkamp.

Scheffler, T. (1996) Worte, Taten, Bilder: Gewaltkult und Realpolitik im palästinensischen Nationalismus. In: Oryval, E./Rao, A./Bollig, M. (Ed.) Krieg und Kampf. Die Gewalt in unseren Köpfen. Berlin: Reimer.

Scheper-Hughes, N./Bourgois, P. (2004) Introduction: Making Sense of Violence. In: Scheper-Hughes, N./Bourgois, P. (Ed.) Violence in War and Peace: An Anthology. Oxford: Wiley-Blackwell.

Schlee, G. (2002) Introduction: Approaches to 'Identity' and 'Hatred': Some Somali and other Perspectives. In: Schlee, G. (Ed.) Imagined Differences. Hatred and the Construction of Identity. Münster: LIT.

Schmidt, B. E./Schröder, I. W. (2001) Introduction: Violent Imaginaries and Violent Practices. In: Schmidt, B. E./Schröder, I. W. (Ed.) Anthropology of Violence and Conflict. London/ New York: Routledge. 
Silverberg, J./Gray, J. P. (Ed.) (1992) Aggression and Peacefulness in Humans and other Primates. New York: Oxford University Press.

Simons, A. (1995) The Beginning of the End. In: Nordstrom, C./Robben, A. (Ed.) Fieldwork under Fire. Contemporary Studies of Violence and Survival. Berkeley/ Los Angeles/ London: University of California Press.

Sluka, J. (2007) Introduction (Section: Fieldwork Conflicts, Hazards, and Dangers). In: Robben, A./Sluka, J. (Ed.) Ethnographic Fieldwork: An Anthropological Reader. Oxford: Blackwell.

Spencer, J. (1993) Violence In: Barnard, A./Spencer, J. (Ed.) Encyclopedia of Social and Cultural Anthropology. London/ New York: Routledge.

Sponsel, L./Gregor T. (Ed.) (1994) The Anthropology of Peace and Nonviolence. Boulder, Col.: Lynne Rienner.

Steward, P. J./Strathern, A. (2002) Violence: Theory and Ethnography. London: Continuum International Publishing Group.

Steward, P. J./Strathern, A. (2004) Witchcraft, Sorcery, Rumors, and Gossip. Cambridge: Cambridge University Press.

Ströbele-Gregor, J. (2004) Das Schweigen brechen: indigene Frauen und häusliche Gewalt - Wandlungsprozesse im Bewusstsein über Menschenrechte in indigenen Gemeinschaften. In: Eckert, J. M. (Ed.) Anthropologie der Konflikte. Georg Elwerts konflikttheoretische Thesen in der Diskussion. Bielefeld: transcript Verlag.

Taussig, M. (1984) Culture of Terror - Space of Death. Roger Casement's Putumayo Report and the Explanation of Torture. In: Comparative Studies in Society and Histo$r y, 26(3): 467-497$.

Traverso, E. (2008) Im Bann der Gewalt. Der europäische Bürgerkrieg 1914-1945. München: Siedler.

Trotha, T. v. (1999) Forms of Martial Power: Total Wars, Wars of Pacification, and Raid. Some Observations on the Typology of Violence. In: Elwert, G./Feuchtwang, S./Neubert, D. (Ed.) Dynamics of Violence. Processes of Escalation and DeEscalation in Violent Group Conflicts. Berlin: Duncker \& Humblot.

Turner, V. W. (1957) Schism and Continuity in an African Society. A Study of Ndembu Village Life. Manchester: Manchester University Press.

Warren, K. B. (2001) Violence in Anthropology. In: Smelser, N. J./Baltes, P. (Ed.) International Encyclopedia of the Social and Behavioral Sciences (Vol. 24). Amsterdam/ Paris/ New York: Elsevier.

Weber, M. (1972) Wirtschaft und Gesellschaft. Tübingen: Paul Siebeck.

Whitehead, N. L. (2004) Introduction: Cultures, Conflicts, and the Poetics of Violent Practice. In: Whitehead, N. L. (Ed.) Violence. Oxford/ Santa Fe, NM: James Currey/School of the American Research Press.

Zitelmann, T. (1991a). Politisches Gemeinschaftshandeln, bewaffnete Gewalt, soziale Mythen: die "Oromo Liberation Front" (OLF) in Äthiopien. In: Scheffler, T. (Ed.) Ethnizität und Gewalt. Hamburg: Deutsches Orient Institut.

Zitelmann, T. (1991b) Refugee Aid, Moral Communities and Resource Sharing. A Prelude to Civil War in Somalia. In: Sociologus, 41(2): 118-138.

Zitelmann, T. (1998) Bomben in Addis Abeba. Nachricht, Gerücht, Selbstinformation. In: Koehler, J./Heyer, S. (Ed.) Anthropologie der Gewalt. Chancen und Grenzen der sozialwissenschaftlichen Forschung. Berlin: VWF Verlag für Wissenschaft und Forschung.

Zitelmann, T. (2003) Somalia, das Horn von Afrika und die Gerüchte vom Einfluss Usama Bin Ladens. In: Meyer, G./Pütz, R./Thimm, A. (Ed.) Terrorismus und Dritte Welt. 
Mainz: Johannes-Gutenberg-Universität (Veröffentlichungen des Interdisziplinären Arbeitskreises Dritte Welt, Bd. 16).

Zitelmann, T. (2004) Wege zur Konfliktethnologie. Eine subjektive Erinnerung. In: Eckert, J. M. (Ed.) Anthropologie der Konflikte. Georg Elwerts konflikttheoretische Thesen in der Diskussion. Bielefeld: transcript Verlag.

Thomas Zitelmann studied Anthropology, Oriental Philology, Islamic Studies and Political Sciences at Frankfurt/Main and at Freie Universität Berlin (FUB). Degrees: MA (1980), Dr. phil. (1991), postdoctoral qualification (2000). Fieldwork in Northeast Africa, the Middle East, Cambodia. Attached senior lecturer at the Institute of Ethnology, FUB. Currently guest professor at FUB.

E-Mail: zitel@zedat.fu-berlin. 